\title{
Three-Dimensional Visualization of Bidirectional Preferential Pathway Conduction of Premature Ventricular Contractions Originating from the Outflow Tract
}

\author{
Seina Yagyu ${ }^{1}$, Koichi Nagashima ${ }^{1}$, Sayaka Kurokawa ${ }^{1}$, Yuji Wakamatsu ${ }^{2}$, Naoto Otsuka ${ }^{1}$, \\ Satoshi Hayashida ${ }^{2}$, Shu Hirata ${ }^{1}$, and Yasuo Okumura ${ }^{1}$ \\ ${ }^{1}$ Nihon University School of Medicine \\ ${ }^{2}$ Nihon University School of Medicine Graduate School of Medicine
}

September 24, 2020

\begin{abstract}
Introduction: Presence of preferential pathway conduction is mostly detected as fractionated presystolic potentials preceding the QRS onset during premature ventricular contractions (PVCs) and late potentials during sinus rhythm (SR), but the electrophysiologic mechanisms and significance of these potentials have not been fully clarified. We describe a PVC case series in which the preferential pathway conduction was 3-dimensionaly visualized by coherent mapping with the conduction velocity vector. Methods: Five PVCs (2 from the left coronary cusp, 2 from the commissure of the left and right coronary cusps, and 1 from the pulmonary artery) in 4 patients for which a fractionated presystolic potential during the PVCs and late potential during SR were recorded at the successful ablation site were reviewed, and 3-dimensional coherent activation maps with the conduction velocity vector during the PVCs and SR were reconstructed. Results: At the successful ablation site, an "M"-shaped discrete presystolic-potential and "W"-shaped discrete late-potential were recorded in all patients. The configuration of the flipped electrogram of the presystolic-potential nearly matched that of the electrogram exhibiting the late-potential. We created coherent activation maps annotating the onset of the presystolic potentials during the PVCs, and the offset of the late potentials during SR, which suggested bidirectional conduction of the preferential pathway connecting the PVC origin to the myocardium. Conclusion: In this case series, the M-shaped presystolic potential and W-shaped late potential were reversely matched. These reverse potentials, and the coherent activation vector maps during PVCs and SR suggested bidirectional conduction of the preferential pathway in opposite directions.
\end{abstract}

\section{Introduction}

Premature ventricular contractions originating from the outflow tract $(\mathrm{OT})$, in particular left ventricular OT (LVOT), often conduct through a preferential pathway to breakout into the global ventricular myocardium. ${ }^{1,2}$ The presence of preferential pathway conduction is mostly detected as fractionated presystolic potentials preceding the QRS onset during PVCs and late potentials during sinus rhythm (SR), and the site with those observations is considered to be an optimal site for a successful ablation. ${ }^{3-5}$ Based on these findings, a radiofrequency (RF) application to the preferential conduction is a well-developed strategy of PVC ablation, but the electrophysiologic mechanisms and significance of presystolic and late potentials during PVCs and SR at the successful ablation site have not been fully clarified. We describe a PVC case series in which the preferential pathway conduction was 3-dimensionaly visualized by coherent mapping with the conduction velocity vector. This case series might add new insight into the mechanisms of preferential pathways and PVCs.

\section{Methods}




\section{Patient characteristics}

Of 51 patients (27 male, $52.4 \pm 17.1$ years) without structural heart disease who underwent catheter ablation of focal PVCs originating from the OT in 2018-2020, 15 cases had LVOT origins and 4 of those patients had PVCs with a fractionated presystolic potentials during the PVCs and late potentials during SR recorded at the successful ablation site. We reviewed these 4 cases using coherent activation maps.

Mapping and ablation were performed using a 3.5-mm-tip catheter (ThermoCool SF; Biosense Webster, Diamond Bar, CA, USA). Coherent activation mapping with the conduction velocity vector during the PVCs was created with the use of an electroanatomic mapping system (CARTO3 system V7, Biosense Webster) with bipolar electrograms, high pass filtered at 20 to $30 \mathrm{~Hz}$ and low pass filtered at $400 \mathrm{~Hz}$. If the presystolic potential was seen, the presystolic potential was manually annotated to its onset. With the use of the mapping points for the PVC, the coherent SR activation map was also created by manual annotation to the offset of the electrograms during the SR beat preceding the PVCs. The late potential was also annotated to its offset. The twelve-lead electrocardiograms and bipolar intracardiac electrograms were recorded with a band pass filter of 30 to $500 \mathrm{~Hz}$ at paper speeds of 100 to $200 \mathrm{~mm} / \mathrm{s}$ and stored on a digital recording system (LabSystem PRO, Bard Electrophysiology, Lowell, MA).

\section{Results}

\section{Case 1}

A 71-year-old man underwent an ablation procedure for PVCs at 35,000 beats/day. The PVCs had a right bundle branch block/inferior axis configuration (Figure 1A). An "M"-shaped discrete presystolic-potential preceding the QRS onset of the PVC by $32 \mathrm{~ms}$ was seen at the right coronary cusp-left coronary cusp commissure, where a "W"-shaped discrete late-potential was recorded during SR (Figure 1B). The configuration of the flipped electrogram of the presystolic-potential nearly matched that of the electrogram exhibiting the late-potential during SR. We created coherent activation maps annotating the onset of the presystolic potentials during the PVC (PVC map, Figure 1C) and offset of the late potentials during SR (SR map, Figure 1D). Those potentials and vector map revealed the bidirectional conduction of the preferential pathway connecting the PVC origin in the right coronary cusp-left coronary cusp (LCC) commissure to the LVOT myocardium. An RF application $(30 \mathrm{~W})$ at that site eliminated both the PVCs and late potential during SR within 4.5 seconds.

\section{Case 2-4}

Similarly, the PVC characteristics in cases 2-4 are shown in the Table and the electrophysiological features in Figures 2-4 including the PVC morphologies (Figures 2A-4A), presystolic potential during the PVC, and late potentials during SR at the successful ablation sites (Figures 2B-4B), and coherent vector activation maps annotating the presystolic potential during the PVC (Figures $2 \mathrm{C}-4 \mathrm{C}$ ) and late potentials during SR (Figures 2D-4D). Bidirectional conduction of the preferential pathways was seen in all cases.

In case 4, 2 different PVCs were targeted: 1 from the PA and the other from the LCC. Bidirectional conduction of the preferential pathway was suggested in both PVCs.

\section{Discussion}

Several previous reports have suggested that these potentials indicate the preferential pathway conduction connecting the origin of an arrhythmia and the myocardial breakout site, and a preferential pathway potential guided ablation can be an effective strategy for PVC ablation. ${ }^{1,3,7}$ We reported that presystolic potentials during PVCs and late potentials during SR were seen at the successful ablation site, and the configuration of the vertically and horizontally-flipped presystolic potentials during PVCs nearly matched that of the late potentials during SR. ${ }^{8}$ In this case series, we also found that the M-shaped presystolic potentials during PVCs and the W-shaped late potentials during SR were reversely matched. These reversed potentials might indicate bidirectional conduction of the preferential pathway in opposite directions. ${ }^{8}$ To demonstrate the hypothesis, we created an activation vector mapping with the annotation of the onset of the presystolic 
potentials and offset of the late potentials. At several points around the successful ablation site, both the presystolic potentials during PVCs and late potentials during SR were observed. At the PVC origin, the presystolic potentials during PVCs were earliest and the offset of the ventricular electrogram during SR was latest. Coherent maps visualizing the vector of this preferential conduction indicated that the propagation direction of the presystolic potentials was opposite to that of the late potentials, which was supportive of our hypothesis.

These potentials are commonly seen in the periaortic area, but rare in the RVOT ${ }^{3}$. In our case series, however, those potentials were also seen in the peri-pulmonary-artery (PA) area. Based on our findings, those $\mathrm{M}$-shaped and $\mathrm{W}$-shaped potentials may reflect the slow conduction due to the smaller amount of myocardium adjacent to the valve annulus such as the aortic valve and pulmonary valve.

The flipped M-shaped presystolic potentials matched the W-shaped late potentials in the majority of our cases, but not in some cases. Those observations might be explained by the difference in the direction between the propagation of the preferential pathway to the ventricular myocardium during the PVC and the propagation of the ventricular myocardium to the preferential pathway during SR. Regarding the association of the catheter position with the direction of the preferential pathway conduction, if the catheter position is not parallel to the direction of the preferential pathway conduction, those potentials might not be reversely matched. To assess the configuration of those potentials, we should consider the catheter position.

\section{Conclusion}

In this case series, the M-shaped presystolic potentials during PVCs and the $\mathrm{W}$-shaped late potentials during SR were reversely matched. Those reversed potentials and the coherent activation vector maps during PVCs and SR suggested bidirectional conduction of the preferential pathway in opposite directions.

\section{References}

1. Yamada T, Murakami Y, Yoshida N, Okada T, Shimizu T, Toyama J, Yoshida Y, Tsuboi N, Muto M, Inden Y, Hirai M, Murohara T, McElderry HT, Epstein AE, Plumb VJ and Kay GN. Preferential conduction across the ventricular outflow septum in ventricular arrhythmias originating from the aortic sinus cusp. $J$ Am Coll Cardiol . 2007;50:884-91.

2. Yamada T, Platonov M, McElderry HT and Kay GN. Left ventricular outflow tract tachycardia with preferential conduction and multiple exits. Circ Arrhythm Electrophysiol . 2008;1:140-2.

3. Hachiya H, Yamauchi Y, Iesaka Y, Yagishita A, Sasaki T, Higuchi K, Kawabata M, Sugiyama K, Tanaka Y, Kusa S, Nakamura H, Miyazaki S, Taniguchi H, Isobe M and Hirao K. Discrete prepotential as an indicator of successful ablation in patients with coronary cusp ventricular arrhythmia. Circ Arrhythm Electrophysiol . 2013;6:898-904.

4. Morita N, Iida T, Akira U and Kobayashi Y. Extensive preferential pathway ablation for the elimination of premature ventricular contractions arising from the right ventricular outflow tract. Indian Pacing Electrophysiol J . 2013;13:88-93.

5. Shirai Y, Goya M, Isobe M and Hirao K. Preferential Pathway Pacing within the Aortic Sinus of Valsalva: Strong Evidence for the Existence of Preferential Conduction with Different Exit Sites Traversing the Ventricular Septum. J Cardiovasc Electrophysiol . 2015;26:805-8.

6. Soejima K, Stevenson WG, Maisel WH, Sapp JL and Epstein LM. Electrically unexcitable scar mapping based on pacing threshold for identification of the reentry circuit isthmus: feasibility for guiding ventricular tachycardia ablation. Circulation . 2002;106:1678-83.

7. Tada H, Naito S, Ito S, Kurosaki K, Ueda M, Shinbo G, Hoshizaki H, Oshima S, Taniguchi K and Nogami A. Significance of two potentials for predicting successful catheter ablation from the left sinus of Valsalva for left ventricular epicardial tachycardia. Pacing Clin Electrophysiol . 2004;27:1053-9. 
8. Yagyu S, Nagashima K, Watanabe R, Arai M, Wakamatsu Y, Otsuka N and Okumura Y. Anterograde and retrograde insulated pathway conduction evidenced by intracardiac electrogram morphologies during premature ventricular contractions and sinus rhythm. HeartRhythm Case Rep . 2019;5:155-158.

Table

\begin{tabular}{|c|c|c|c|c|c|}
\hline Case & 1 & 2 & 3 & $4-1$ & $4-2$ \\
\hline Age & 71 & 58 & 69 & 13 Male $64 \%$ & 13 Male $64 \%$ \\
\hline Sex & Male & Male & Female & & \\
\hline LVEF (\%) & $69 \%$ & $57 \%$ & $64 \%$ & & \\
\hline $\begin{array}{l}\text { PVC number } \\
\text { (/day) }\end{array}$ & 35,000 & 90,000 & 15,000 & 74,000 & 74,000 \\
\hline PVC origin & $\begin{array}{l}\text { LCC-RCC } \\
\text { comissure }\end{array}$ & $\begin{array}{l}\text { LCC-RCC } \\
\text { comissure }\end{array}$ & LCC & $\mathrm{PA}$ & LCC \\
\hline $\begin{array}{l}\text { PVC } \\
\text { morphology }\end{array}$ & $\mathrm{RBBB}$ & $\mathrm{RBBB}$ & $\mathrm{RBBB}$ & $\mathrm{RBBB}$ & $\mathrm{RBBB}$ \\
\hline PVC axis & Inferior & Inferior & Inferior & Inferior & Inferior \\
\hline $\begin{array}{l}\text { Transitional } \\
\text { zone }\end{array}$ & $<\mathrm{V} 1$ & $<\mathrm{V} 1$ & $<\mathrm{V} 1$ & V1 & V1 \\
\hline $\begin{array}{l}\text { EGM-QRS } \\
\text { interval }(\mathrm{ms})\end{array}$ & 32 & 20 & 40 & 28 & 24 \\
\hline
\end{tabular}

EGM, electrogram; LBBB, left bundle branch block; LCC, left coronary cusp; LVEF, left ventricular ejection fraction; PA, pulmonary artery; PVC, premature ventricular contraction; RBBB, right bundle branch block; RCC, right coronary cusp; SHD, structural heart disease.

\section{Figure legends}

Figure 1. Twelve-lead electrocardiogram of a PVC recorded during the procedure (A) . Intracardiac electrograms at the successful ablation site. A presystolic potential during the PVC and late potential during SR are recorded by the ablation catheter. The late potential during SR was eliminated after the successful ablation(B). Coherent vector activation maps annotating the presystolic potentials during the PVC (C) and late potentials during SR(D). The ablation catheter at the successful ablation site is shown in the figure.

SR, sinus rhythm; PVC, premature ventricular contraction; LCC, left coronary cusp; RCC, right coronary cusp; ABL, ablation.

Figure 2-4.

Similarly, a twelve-lead electrocardiogram of a PVC obtained during the procedure (2A-4A), intracardiac electrograms recorded at the successful ablation site (2B-4B), and Coherent vector activation maps (2C4C, 2D-4D).

\section{Hosted file}

Figure 9.12.14.pptx available at https://authorea.com/users/361261/articles/482721-threedimensional-visualization-of-bidirectional-preferential-pathway-conduction-of-prematureventricular-contractions-originating-from-the-outflow-tract 